\title{
Multisegment Multiconductor Transmission Line Model of High-Current Card-Edge Connectors With Analytically Calculated Model Parameters
}

Raul Blecic, Student Member, IEEE, Niko Bako, Renaud Gillon, Member, IEEE, Bart Nauwelaers, Senior Member, IEEE, and Adrijan Baric, Senior Member, IEEE

\begin{abstract}
R. Blecic is with the ESAT-TELEMIC, Katholieke Universiteit Leuven, Heverlee B-3001, Belgium, and also with the Faculty of Electrical Engineer-ing and Computing, University of Zagreb, Zagreb 10000, Croatia (e-mail:, raul.blecic@fer.hr).

N. Bako was with the Faculty of Electrical Engineering and Computing, University of Zagreb, Zagreb 10000, Croatia. He is now with Texas Instruments Deutschland GmbH, Freising D-85356, Germany (e-mail:n-bako@ti.com).

R. Gillon is with the ON Semiconductor, Oudenaarde 9700, Belgium (e-mail:, Renaud.Gillon@onsemi.com).

B. Nauwelaers is with the Katholieke Universiteit Leuven, Heverlee B-3001, Belgium (e-mail:,Bart.Nauwelaers@esat.kuleuven.be).

A. Baric is with the Faculty of Electrical Engineering and Computing, Uni-versity of Zagreb, Zagreb 10000, Croatia (email:,adrijan.baric@fer.hr).

Color versions of one or more of the figures in this paper are available online at http://ieeexplore.ieee.org.
\end{abstract}

\begin{abstract}
Modeling of two high-current card-edge connectors is presented. The modeling methodology consists of dividing the connectors into uniform segments and modeling each segment by a multiconductor transmission line. The calculation of the per-unit-length parameters of each segment is based on the analytic expression for a generalized capacitance matrix of widely sepa-rated conductors. The proposed modeling methodology generates the circuit model solely from the geometrical parameters of the con-nectors without the need to perform measurements and/or electro-magnetic simulations. The modeling methodology is applied to two 62-pin high-current card-edge connectors that are used in the mea-surement setup for characterization of radiated electromagnetic emission of switched-mode power converters. Two printed circuit boards are designed to characterize the connectors and to vali-date the modeling methodology. The model of the complete char-acterization setup shows good agreement with the $S$ parameter measurements up to $3 \mathrm{GHz}$.
\end{abstract}

\section{INTRODUCTION}

Connectors are used in many electronic systems for various applications, mainly to add versatility. Impedancemismatch and discontinuities introduced by the connectors can have a significant impact on electromagnetic compatibility

(EMC) and signal integrity of the system [1], [2]. Considering a constant increase of integration densities, having a possibility of performing fast and accurate system-level simulations in an early design stage and optimizing its performance is of increasing importance.

Connectors are typically modeled by multisegment lumpedelements as in [1], [3]-[7] or multisegment transmission lines as in [8]-[10]. In all of these papers, the model parameters are fitted to the measured data, either the S-parameter measurements as in [3], the time-domain reflectometry
(TDR) as in [4], [6]-[9], or both as in [1] and [5].

Alternatively, electromagnetic (EM) simulations can be used to generate the required data [10], [11]. Modeling based on the measurements and/or EM simulations is time-consuming. Moreover, it requires a specially designed characterization setup, which does not necessarily correspond to the setup in which the connector will be used.

In this paper, a connector is divided into uniform segments that are modeled by multiconductor transmission lines (MTLs). The calculation of the transmission line capacitance and inductance matrices of each segment is based on the analytic expression for the generalized capacitance matrix of widely separated conductors [12], [13], which is applied to the modeling of ribbon cables in [13] and [14]. The result of the modeling methodology is a circuit model generated solely from the geometrical parameters of the connector without the need to perform measurements and/or EM simulations. The generation of the model is fast and simple, which allows easy model parameterization. The circuit implementation ensures short simulation times. The methodology can be applied to any number of pins and the coupling between all the pins is inherently taken into account. These features of the proposed modeling methodology enable fast analysis of EMC of complex systems connected by the connectors.

The modeling methodology is applied to two 62-pin card-edge connectors. The usage of the two connectors is proposed in [15] to enable measurements of the magnetic field component normal to the printed circuit board (PCB) plane. The connectors are used in [16] and [17] to characterize the radiated EM emission of two synchronous buck converters. The purpose of the connectors is to position a converter inside the transverse electromagnetic (TEM) cell in multiple, well-defined orientations relative to the septum of the TEM cell, and to provide the control signals and the supply and load to the converters. With the current rating of $3 \mathrm{~A}$ per pin, the connectors enable 
characterization of EMC of switched-mode power converters operating at high current levels.

This paper is structured as follows. Section II presents the model of the connectors and applies the modeling methodology to two 62-pin high-current card-edge connectors. Section III presents the characterization setup and its model. Section IV compares the simulation results to the measurements. Section V concludes the paper.

\section{MODEL OF THE CONNECTOR}

\section{A. Modeling Methodology}

A connector is divided into segments that are uniform. Each segment is treated as a lossless MTL. The per-unit-length (p.u.1.) parameters of each segment are derived from the analytic expression for the $p \times p$ p.u.l. generalized capacitance matrix of $p$ widely separated conductors. The model of the connector is built by cascading the MTLs of all segments.

The generalized capacitance matrix is the p.u.l. parameter matrix in which the potentials of all conductors are referenced to infinity [12]. It is given by [12], [13]

$$
\mathscr{C}^{-1}=\frac{-1}{2 \pi \epsilon_{r} \epsilon_{0}}\left[\begin{array}{cccc}
\ln \left(r_{1}\right) & \ln \left(d_{21}\right) & \ldots & \ln \left(d_{p 1}\right) \\
\ln \left(d_{21}\right) & \ln \left(r_{2}\right) & \ln \left(d_{23}\right) & \ldots \\
\vdots & & \ddots & \vdots \\
\ln \left(d_{p 1}\right) & & \ldots & \ln \left(r_{p}\right)
\end{array}\right]
$$

where $\epsilon_{r}$ is the relative permittivity of the surrounding material, $\epsilon_{0}$ is the permittivity of free space, $r_{i}$ is the radius of $i$ th circular pin, and $d_{i j}$ is the center-to-center distance between the $i$ th and $j$ th $\operatorname{pin}(i, j=1, \ldots, p)$. The wide-separation approximation is valid if the ratio of the pin separation to the pin radius is greater than or equal to 4 [12], [13], which is true for the modeled connectors.

The p.u.l. capacitance matrix is calculated from the p.u.l. generalized capacitance matrix as follows.

1) The pins are divided into $s$ signal pins and $(p-s)$ reference pins. The two sets depend on the actual application of the connector (i.e., on the function of each pin). All the reference pins are assumed to be at the same (reference) potential.

2) Matrix $\mathscr{C}$ is rearranged so that the first $s$ rows and columns correspond to the signal pins, while the last $(p-s)$ rows and columns correspond to the reference pins.

3) The $s \times s$ p.u.l. transmission line capacitance matrix $\mathbf{C}$ is calculated from the $p \times p$ p.u.l. generalized capacitance matrix $\mathscr{C}$ by [12], [13]

$$
C_{i j}=\frac{\mathscr{C}_{i j}\left(M_{0}-M_{i}-M_{j}-\mathscr{C}_{i j}\right)-M_{i} M_{j}}{M_{0}}
$$

where $M_{0}$ is the sum of all terms of $\mathscr{C}, M_{i}$ is the sum of all terms in the $i$ th row of $\mathscr{C}$ except $\mathscr{C}_{i j}, M_{j}$ is the sum of all terms in the $j$ th column of $\mathscr{C}$ except $\mathscr{C}_{i j}$, while $i$ and $j$ are the row and column indices of the signal pins, respectively $(i, j=1, \ldots, s)$.

The $s \times s$ p.u.l. transmission line inductance matrix is calculated from the $s \times s$ p.u.l. transmission line capacitance matrix

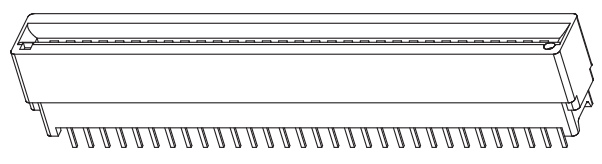

(a)

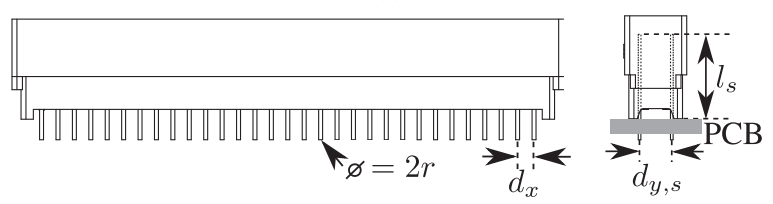

(b)

(c)

Fig. 1. Straight 62-pin high-current card-edge connector. (a) Threedimensional view. (b) Front view. (c) Side view.

TABLE I

GeOMetrical Parameters of THE Modeled Straight 62-PIN High-CurRENT CARD-EDGE CONNECTOR

\begin{tabular}{llc}
\hline Description & Parameter & Value \\
\hline Separation between two adjacent pins in a row & $d_{x}[\mathrm{~mm}]$ & 2.54 \\
\hline Separation between two rows of pins & $d_{y, s}[\mathrm{~mm}]$ & 4.85 \\
\hline Length of the pins above the PCB & $l_{s}[\mathrm{~mm}]$ & 12 \\
\hline Radius of the pins & $r[\mathrm{~mm}]$ & 0.254 \\
\hline Relative permittivity of the surrounding material & $\epsilon_{r, s}$ & 4.2 \\
\hline
\end{tabular}

as [12], [13]

$$
\mathbf{L}=\mu_{0} \epsilon_{0} \mathbf{C}_{0}^{-1}
$$

where $\mathbf{C}_{0}$ is the $s \times s$ p.u.l. transmission line capacitance matrix calculated by (2) for the same structure, but with the dielectric material removed (the relative permittivity of the surrounding material is set to $\epsilon_{r}=1$ ).

\section{B. Model of the 62-Pin High-Current Card-Edge Connectors}

1) Straight Connector: The straight 62-pin high-current card-edge connector [18] is shown in Fig. 1. It consists of two parallel rows of pins (31 pins per row). Each pin is $12 \mathrm{~mm}$ long above the backplane (the PCB on which the connector is mounted, see Fig. 1), i.e., $l_{s}=12 \mathrm{~mm}$. The pins are approximated to have a circular cross section. The surrounding material is approximated as homogeneous with the relative permittivity equal to that of the housing (i.e., the finite dimensions of the housing are not taken into account).

Under the aforementioned approximations, the complete geometry of the straight connector can be considered as uniform, and it is modeled by one MTL. Its geometrical parameters are given in Table I. The dimensions in Table I show that $d_{x} / r>4$, which was the assumption of wide separation between pins used in the derivation of $\mathscr{C}$ in (1). The generalized capacitance matrix is calculated by (1) and it results in a $62 \times 62$ matrix.

The checkerboard pinout shown in Fig. 2 is used for the characterization of the connectors and for the validation of the model. Every second pin is used as a signal pin, which yields altogether 31 signal pins and 31 reference pins. The transmission capacitance and inductance matrices $\mathbf{C}$ and $\mathbf{L}$ are calculated from the generalized capacitance matrix by (2) and (3), respectively, 


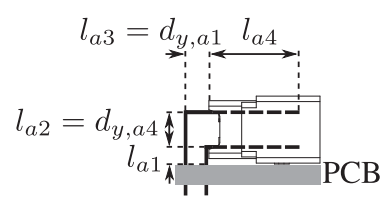

(a)

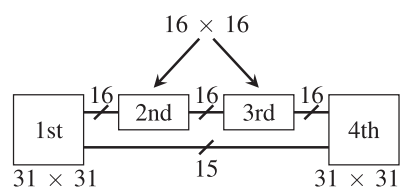

(b)
Fig. 2. Angled 62-pin high-current card-edge connector. (a) Side view with four uniform segments indicated. (b) Schematic representation of cascading the four segments.

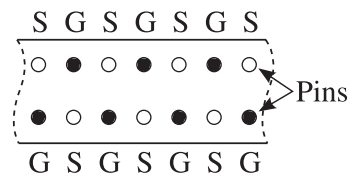

Fig. 3. Checkerboard pinout used for the characterization of the connectors and for the validation of the model. The signal pins are marked by $\mathrm{S}$ (open circles), while the reference pins are marked by $\mathrm{G}$ (black dots).

\section{TABLE II}

GEOMETRICAL PARAMETERS OF THE MODELED ANGLED 62-PIN HIGH-CURRENT CARD-EDGE CONNECTOR

\begin{tabular}{llc}
\hline Description & Parameter & Value \\
\hline Separation between two adjacent pins in a row & $d_{x}[\mathrm{~mm}]$ & 2.54 \\
\hline $\begin{array}{l}\text { Separation between two rows of pins for the } \\
\text { first segment }\end{array}$ & $d_{y, a 1}[\mathrm{~mm}]$ & 3.81 \\
$\begin{array}{l}\text { Separation between two rows of pins for the } \\
\text { fourth segment }\end{array}$ & $d_{y, a 4}[\mathrm{~mm}]$ & 4.85 \\
\hline \begin{tabular}{l} 
Length of the pins in the first segment \\
\hline $\begin{array}{l}\text { Length of the pins in the second segment } \\
\text { Length of the pins in the third segment }\end{array}$
\end{tabular} & $l_{a 2}[\mathrm{~mm}]$ & 2.2 \\
\hline $\begin{array}{l}\text { Length of the pins in the fourth segment } \\
l_{a 3}[\mathrm{~mm}]\end{array}$ & $l_{a 4}[\mathrm{~mm}]$ & 3.81 \\
\hline $\begin{array}{l}\text { Radius of the pins } \\
\text { Relative permittivity of the surrounding } \\
\text { material for the first three segments }\end{array}$ & $\epsilon_{r, a 1,2,3}$ & $1 \mathrm{~mm}]$ \\
\hline $\begin{array}{l}\text { Relative permittivity of the surrounding } \\
\text { material for the fourth segment }\end{array}$ & $\epsilon_{r, a 4}$ & 0.254 \\
\hline
\end{tabular}

and they are $31 \times 31$ matrices. The pinout will be different for different applications; however, the same methodology can be used to generate the model of the connector regardless of the pinout used.

2) Angled Connector: The angled 62-pin high-current card-edge connector [19] is shown in Fig. 3. It consists of two rows of pins (31 pins per row). The pins closer to the backplane are shorter. The connector can be divided into four uniform segments. Both rows of pins are present in the first and fourth segments, while only the longer pins are present in the second and third segments. The first three segments are approximated to be completely surrounded by air. The fourth segment is the same as the straight connector.

The angled connector is modeled by four MTLs that corre-spond to the four segments. The geometrical parameters of each segment are given in Table II. The generalized capacitance ma-trix is calculated by (1) and it results in a $62 \times 62$ matrix for the first and fourth segments, and in a $31 \times 31$ matrix for the second and third segments.

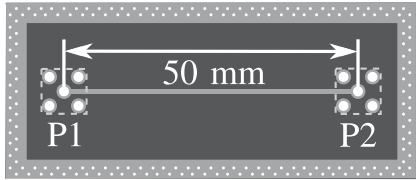

(a)

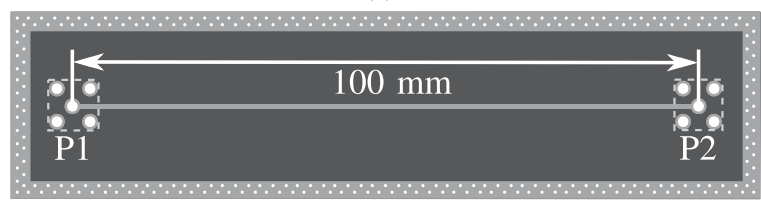

(b)

Fig. 4. (a) $50 \mathrm{~mm}$ long, $0.65 \mathrm{~mm}$ wide, $50-\Omega$ microstrip line calibration structure. (b) $100 \mathrm{~mm}$ long, $0.65 \mathrm{~mm}$ wide, $50-\Omega$ microstrip line calibration structure. Light gray color represents the first layer, dark gray color represents the second, third, and fourth layers (which are all the same), while white holes are the through-hole vias.

The same checkerboard pinout is used as for the straight connector. The 31 signal pins and 31 reference pins are present in the first and fourth segments, while 16 signal pins and 15 reference pins are present in the second and third segments. The transmission line capacitance and inductance matrices $\mathbf{C}$ and $\mathbf{L}$ are calculated from the generalized capacitance matrix by (2) and (3), respectively, and they are $31 \times 31$ matrices for the first and fourth segments, while they are $16 \times 16$ matrices for the second and third segments. The four segments are cascaded to complete the model of the connector, as schematically shown in Fig. 3(b).

The wide separation approximation is valid for both connec-tors since the ratio of the smallest pin separation to the pin radius is 2.54 $\mathrm{mm} / 0.254 \mathrm{~mm}=10>4$. The transmission capacitance and inductance matrices $\mathbf{C}$ and $\mathbf{L}$ are implemented in a circuit simulator by a W-element [20].

\section{MODEL OF THE CHARACTERIZATION SETUP}

The characterization setup is designed to validate the mod-els of the two modeled connectors. The setup consists of two PCBs (the backplane and the characterization board). Addi-tionally, two calibration structures are designed to extract the relative permittivity of the PCB substrate and to optimize the parameters of the model of the subminiature version A (SMA) connectors [21]. Knowing the characteristics of the substrate and that of the SMA connectors allows generation of the model of the complete setup and its comparison to the measurements.

\section{A. Calibration Structures}

The calibration structures are $50 \mathrm{~mm}$ and $100 \mathrm{~mm}$ long, $0.65 \mathrm{~mm}$ wide, and $50-\Omega$ microstrip lines that are used to connect two SMA connectors $\mathrm{P} 1$ and $\mathrm{P} 2$, as shown in Fig. 4. The struc-tures are designed on four-layer PCBs. The first layer is a signal layer, while the second, third, and fourth are the reference lay-ers. The four-layer PCB stack is used to allow a simple design of calibration structures, backplane, and DUT board. The parame-ters of the PCBs are given in Table III. The relative permittivity of the PCB substrate is extracted from the measured phase dif-ference of the transmission characteristics of the two calibration 
TABLE III

PARAMETERS OF THE PCBS

\begin{tabular}{lll}
\hline Description & Parameter & Value \\
\hline Substrate thickness (1st-to-2nd layer) & $h_{1,2}[\mathrm{~mm}]$ & 0.36 \\
\hline Substrate thickness (2nd-to-3rd layer) & $h_{2,3}[\mathrm{~mm}]$ & 0.71 \\
\hline Substrate thickness (3rd-to-4th layer) & $h_{3,4}[\mathrm{~mm}]$ & 0.36 \\
\hline Relative permittivity of the substrate at $1 \mathrm{GHz}$ & $\epsilon_{r, s u b}$ & 4.82 \\
\hline Tangent delta of the substrate & $\tan \delta$ & 0.02 \\
\hline Copper plate thickness & $t_{C u}[\mathrm{~mm}]$ & 0.035 \\
\hline Copper conductivity & $\sigma_{C u}[\mathrm{~S} / \mathrm{m}]$ & $4.1 \mathrm{e} 7$
\end{tabular}

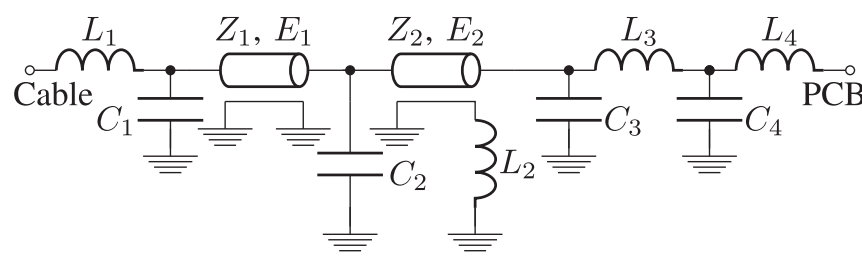

Fig. 5. Circuit model of an SMA connector [21]
TABLE IV
OPTIMIZED PARAMETERS OF THE CIRCUIT MODEL OF THE SMA CONNECTORS [21]

\begin{tabular}{llc}
\hline Description & Parameter & Value \\
\hline Cable-connector transition: & & \\
LC stub inductance & $L_{1}[\mathrm{pH}]$ & 179.2 \\
LC stub capacitance & $C_{1}[\mathrm{fF}]$ & 37.8 \\
\hline Coaxial part of the connector: & & \\
transmission line (TL) characteristic impedance & $Z_{1}[\Omega]$ & 50.5 \\
TL electrical length (at 1 GHz) & $E_{1}[\mathrm{deg}]$ & 10.03 \\
\hline Capacitance of the air gap between the & $C_{2}[\mathrm{fF}]$ & 117.1 \\
connector and the substrate & & \\
Connector pins: & $Z_{2}[\Omega]$ & 47.4 \\
TL characteristic impedance & $E_{2}[\mathrm{deg}]$ & 2.97 \\
TL electrical length (at $1 \mathrm{GHz})$ & $L_{2}[\mathrm{pH}]$ & 0.1 \\
\hline Ground plane inductance & & \\
\hline Right-angle discontinuity: & $L_{3}[\mathrm{pH}]$ & 0.6 \\
LC stub inductances & $L_{4}[\mathrm{pH}]$ & 0.5 \\
& $C_{3}[\mathrm{fF}]$ & 681.0 \\
LC stub capacitances & $C_{4}[\mathrm{fF}]$ & 167.4 \\
\hline
\end{tabular}

structures having different lengths by the two-microstrip-line method [22].

The model of each calibration structure consists of the mi-crostrip line, which is modeled in Keysight ADS Momen-tum [23], and two SMA connector models [21]. The parameters of the SMA connector model are optimized to fit the measured transmission $\left(S_{12}, S_{21}\right)$ and reflection characteristics $\left(S_{11}, S_{22}\right)$ of the calibration structures.

The model of the SMA connector is shown in Fig. 5. Its op-timized parameters and their description are given in Table IV. The model parameters related to the cable-to-connector transi-tion $\left(L_{1}, C_{1}\right)$, coaxial part of the connector $\left(Z_{1}, E_{1}\right)$, capacitance of the air gap $\left(C_{2}\right)$, and connector pins $\left(Z_{2}, E_{2}\right)$ are considered

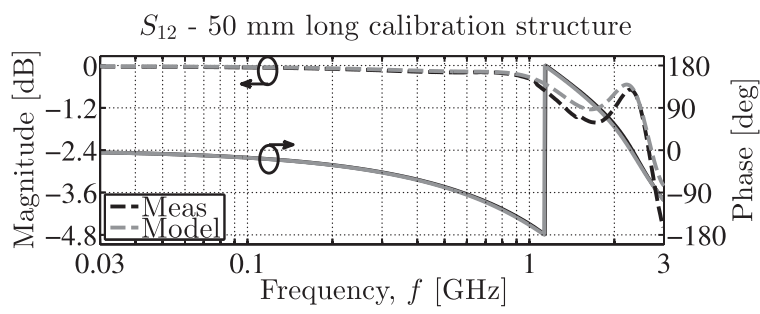

(a)

$S_{11}-50 \mathrm{~mm}$ long calibration structure

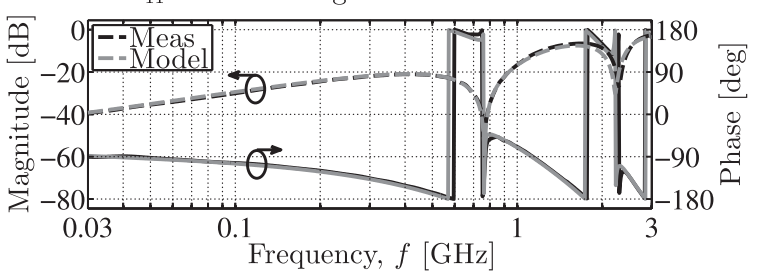

(b)

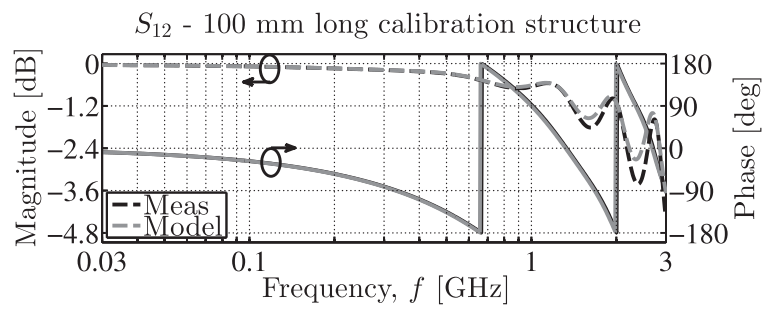

(c)

$S_{11}-100 \mathrm{~mm}$ long calibration structure

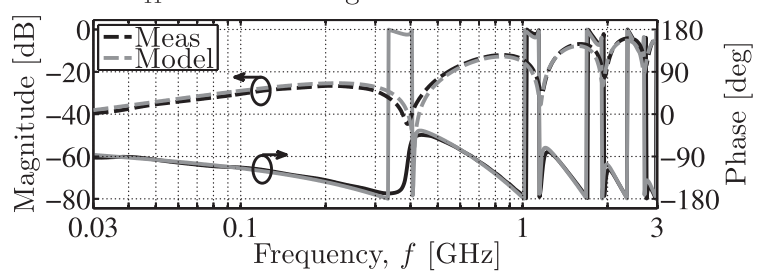

(d)

Fig. 6. $S$-parameters of the calibration structures. (a) Transmission $S 12$ of the 50 -mm-long calibration structure. (b) Reflection $S 11$ of the 50-mm-long calibration structure. (c) Transmission $S 12$ of the 100 -mm-long calibration structure. (d) Reflection $S_{11}$ of the $100-\mathrm{mm}$ long calibration structure.

to be independent of the PCB on which the SMA connectors are mounted and these parameters are allowed to vary $\pm 10 \%$ from the values published in [21]. Those parameters that are depen-

dent on the PCB $\left(L_{2}, L_{3}, L_{4}, C_{3}, C_{4}\right)$ are allowed to change in a wider range. The obtained values of the capacitances $C_{3}$ and $C_{4}$ are larger, while those of inductances $L_{2}, L_{3}$, and $L_{4}$ are smaller than that from [21]. This correlates well with the smaller thickness of the substrate between the signal and the closest reference layer when compared to the thicknesses used in [21].

The comparison of the measured and modeled characteristics of the calibration structures is shown in Fig. 6. The modeled and the measured characteristics show very good agreement in the frequency range up to $3 \mathrm{GHz}$.

\section{B. Backplane}

Backplane is a four-layer, $100 \mathrm{~mm} \times 100 \mathrm{~mm}$ PCB designed according to the TEM cell measurement standard [24], with 


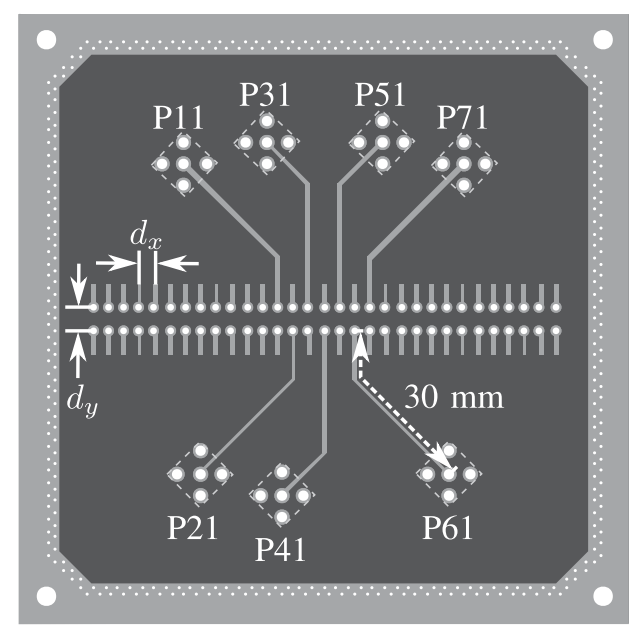

Fig. 7. Backplane for the characterization of the connector. Light gray color represents the first layer, dark gray represents the second, third, and fourth layers (which are all the same), while white holes are the through-hole pads.

the 62-pin connectors mounted in the middle of the PCB. The first layer is the signal layer, while the second, third, and fourth are the reference layers. The top view of the PCB is shown in Fig. 7. The parameters of the PCB are the same as those used for the calibration structures (see Table III). The designed pinout is the checkerboard pinout (see Fig. 2). Every second through-hole pad is connected to the reference planes in the second, third, and fourth layers. Three signal pads on one side and four on the other side of the 62-pin connectors are con-nected to the SMA connectors by $30 \mathrm{~mm}$ long, $0.65 \mathrm{~mm}$ wide, and $50-\Omega$ microstrip feed lines. Other signal and reference pins are connected to the 3.81-mm-long microstrip lines that are left open.

The model of the backplane consists of seven SMA connec-tors, 31 capacitances of the through-hole pads (one for each signal pad), and coupled microstrip lines simulated in Keysight ADS Momentum [23]. The simulation domain in Momentum consists of all 62 microstrip lines that are present on the back-plane. The 31 lumped ports are placed, one at each signal pad of the 62-pin connectors, while 7 lumped ports are placed at the central pad of each SMA connector. The simulation results in a $38 \times 38 S$-parameter matrix.

The capacitances of the signal pads are taken into account by the analytical expression described in [25] and summarized as follows. The coaxial capacitance between the pad and the plate, due to the finite thickness of the plate, is calculated as [25]

$$
C_{a}=\frac{2 \pi \epsilon_{r, \mathrm{sub}} \epsilon_{0} t_{\mathrm{Cu}}}{\ln (b / a)}
$$

where $\epsilon r$, sub is the relative permittivity of the PCB substrate, $\epsilon_{0}$ is the permittivity of free space, $t \mathrm{Cu}$ is the copper plate thickness, while $a$ $=0.55 \mathrm{~mm}$ and $b=1.1 \mathrm{~mm}$ are the radii of the pad and the antipad, respectively. The outer boundary of the pad [25] is placed at the half-distance between the two closest pads $R=1.27 \mathrm{~mm}$ (see Fig. $8)$.

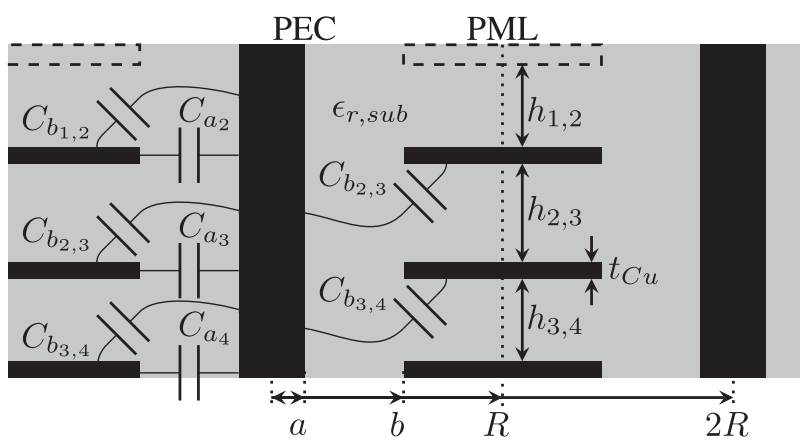

Fig. 8. Cross section of the backplane and the through-hole pad with the ca-pacitances of the pad model indicated (PEC represents perfect electric conductor and PML represents perfectly matched layer).

The capacitance between the through-hole pad barrel and the plate is calculated as [25]

$$
\begin{aligned}
C_{b}= & \frac{8 \pi \epsilon_{r, \mathrm{sub}} \epsilon_{0}}{h_{u, u+1} \ln (b / a)} \sum_{n=1,3,5, \ldots}^{2 N-1} \frac{\left(1-\Gamma_{a}^{(n)} \Gamma_{R}^{(n)}\right)^{-1}}{k_{n}^{2} H_{0}^{(2)}\left(k_{n} a\right)} \\
& \cdot\left\{\left[H_{0}^{(2)}\left(k_{n} b\right)-H_{0}^{(2)}\left(k_{n} a\right)\right]\right. \\
& \left.+\Gamma_{R}^{(n)}\left[J_{0}\left(k_{n} b\right)-J_{0}\left(k_{n} a\right)\right]\right\}
\end{aligned}
$$

where $h_{u, u+1}$ is the thickness of the substrate between the $u$ th and $(u+1)$ th copper layer $(u=1,2,3), H_{0}^{(2)}$ is the zeroth-order Hankel function of the first kind, $J_{0}$ is the zeroth-order Bessel function of the first kind, $\Gamma_{a}^{(n)}$ is the reflection coefficient at the pad boundary and it is modeled as a PEC [25]

$$
\Gamma_{a}^{(n)}=-\frac{J_{0}\left(k_{n} a\right)}{H_{0}^{(2)}\left(k_{n} a\right)} .
$$

$\Gamma_{R}^{(n)}$ is the reflection coefficient at the outer boundary $R$. Since $R / h_{u, u+1}>1.7$ for all three substrate thicknesses of the backplane, the outer boundary is modeled as a PML [25]

$$
\Gamma_{R}^{(n)}=0
$$

$k_{n}$ is the radial wavenumber

$$
k_{n}=\sqrt{k_{0}^{2} \epsilon_{r, \mathrm{sub}}-\left(\frac{n \pi}{h_{u, u+1}}\right)^{2}}
$$

$k_{0}=2 \pi / \lambda$ is the wavenumber, $\lambda=c / f$ is the wavelength, $c$ is the speed of light in free space, $f$ is the frequency, $n$ is the higher mode index, while $2 N$ is the total number of higher modes taken into account $(2 N$ $=60)$.

Assuming that all three reference layers are at the same (reference) potential, the total capacitance of the through-hole pad is calculated as

$$
C_{\mathrm{pad}}=C_{a_{2}}+C_{a_{3}}+C_{a_{4}}+C_{b_{1,2}}+2 C_{b_{2,3}}+2 C_{b_{3,4}} .
$$

Three capacitances $C_{a}$ are present in (9), one for each refer-ence layer of the backplane. Five capacitances $C_{b}$ are present, one for a part of the pad barrel referenced to each reference plane. Since the part of the barrel between the first and the sec-ond layers is referenced to the second layer only, its capacitance 
TABLE V

Calculated Through-Hole Pad Capacitances $(a=0.55 \mathrm{MM}$, $b=1.1 \mathrm{MM}, 2 N=60)$

\begin{tabular}{llc}
\hline Description & Parameter & Value \\
\hline Coaxial capacitance & $C_{a_{2}}=C_{a_{3}}=C_{a_{4}}[\mathrm{fF}]$ & 13 \\
\hline $\begin{array}{l}\text { Capacitance between the pad } \\
\text { barrel and the 2nd layer }\end{array}$ & $C_{b_{1,2}}[\mathrm{fF}]$ & 66 \\
\hline $\begin{array}{l}\text { Capacitance between the pad } \\
\text { barrel and the 3rd layer }\end{array}$ & $C_{b_{2,3}}[\mathrm{fF}]$ & 124 \\
$\begin{array}{l}\text { Capacitance between the pad } \\
\text { barrel and the 4th layer }\end{array}$ & $C_{b_{3,4}}[\mathrm{fF}]$ & 66 \\
\hline Total pad capacitance & $C_{p a d}[\mathrm{fF}]$ & 485 \\
\hline
\end{tabular}

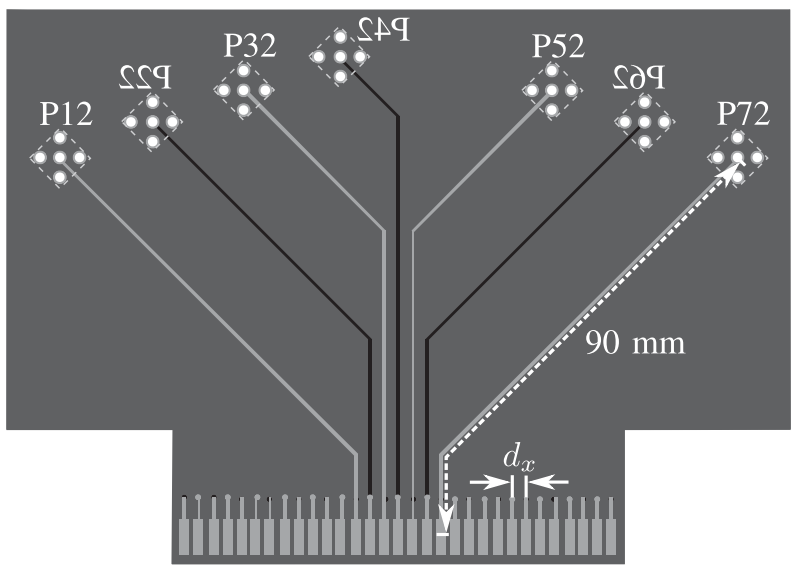

Fig. 9. Characterization board for the characterization of the connector. Light gray color represents the first layer, dark gray represents the second and the third layers (which are the same), black represents the fourth layer, while white holes are the through-hole vias.

$C_{b 1}, 2$ is present only once. The part of the barrel between the second and the third layers is referenced to two layers, so its capacitance $\mathrm{Cb}_{2}$ is present twice (see Fig. 8). The capacitance, 3 $\mathrm{Cb}_{3}$ is present twice for the same reason. The capacitance $C_{b}, 4$ is frequency dependent; however, its dependence is weak in the frequency range of interest, and it is modeled by a constant value. The calculated pad capacitances are given in Table $\mathrm{V}$.

\section{Characterization Board}

Characterization board is a four-layer PCB that is inserted into the connector opening. Its top view is shown in Fig. 9. The parameters of the PCB are the same as those used for the calibration structures and backplane (see Table III). The first and fourth layers are signal layers, while the second and third are reference layers. Every second pad on the first and fourth layers is connected to the reference planes on the second and third layers by a 3.81-mm-long microstrip line and a blind via of the radius equal to $0.25 \mathrm{~mm}$. Four $90 \mathrm{~mm}$ long, $0.65 \mathrm{~mm}$ wide, $50-\Omega$ microstrip feed lines on the first layer and three on the fourth layer connect the 62-pin connectors to the SMA connectors. All other signal pins are connected to 3.81-mm-long microstrip lines that are left open, similarly to the backplane (see Fig. 7).

The model of the characterization board consists of seven SMA connectors and coupled microstrip lines simulated in
Keysight ADS Momentum [23]. The simulation domain in Momentum consists of all 62 microstrip lines that are present on the characterization board. The 31 lumped ports are placed at the pads that correspond to the signal pins of the 62-pin connectors, while 7 lumped ports are placed at the central pad of each SMA connector. The simulation results in a $38 \times 38$ matrix. Similarly to the backplane, both reference layers are assumed to be at the same (reference) potential and they are modeled as ideal reference planes.

\section{Model of the Complete Characterization Setup}

The schematic representation of the model of the setup is shown in Fig. 10. The model consists of the backplane (the SMA connectors, the coupled microstrip feed lines, and the capacitances of the pads), the proposed model of the connector implemented by cascaded W-elements (one W-element is used for the straight connector, while four cascaded W-elements are used for the angled connector), and the characterization board (the coupled microstrip feed lines and the SMA connectors).

Simulation of the proposed model of the connectors in 21 fre-quency points takes $3 \mathrm{~s}$ on a desktop computer with $8 \mathrm{~GB}$ of memory and a 2.66-GHz quad-core processor, for each connec-tor. For comparison, simulations of the same structures in the commercial EM solver on the same computer, with the mini-mal memory target mesh option [26], take 26 minutes for the straight connector (out of which $10 \mathrm{~min}$ for the mesh and $46 \mathrm{~s}$ per frequency point) and $34 \mathrm{~min}$ for the angled connector (out of which 16 min for the mesh, and $52 \mathrm{~s}$ per frequency point). Once the model of each element of the characterization setup is obtained, the simulation of the complete characterization setup with 400 points per decade takes $5 \mathrm{~min}$, on the same computer, for each connector.

\section{RESULTS}

The characterization setup allows the characterization of the transmission from the backplane, through the connector, to the characterization board (P41P42), the crosstalk to the first (P41P31), and to the second neighbor on the backplane side (P41P21), i.e., the near-end crosstalk (NEXT), and the crosstalk to the first (P41P52) and to the second neighbor on the character-ization board side (P41P62), i.e., the far-end crosstalk (FEXT). The measurements are performed by a 2-port VNA. While one set of the measurements is performed (e.g., the transmission P41P42), all other backplane and characterization board SMA connectors are terminated into $50 \Omega$ [27]. The comparison of the modeled and measured characteristics for the straight and angled connector is shown in Figs. 11 and 12, respectively. The characteristics of the model of the charac-terization setup when each pin of the connector is modeled by a short is added to the graphs to emphasize the impact of the connectors.

The feature selective validation (FSV) technique [28]-[30] is applied to the measured and simulated data to get a numeri-cal level of agreement between them. The amplitude difference measure (ADM) compares amplitudes and trends of the two data sets, the feature difference measure (FDM) compares the rapidly 


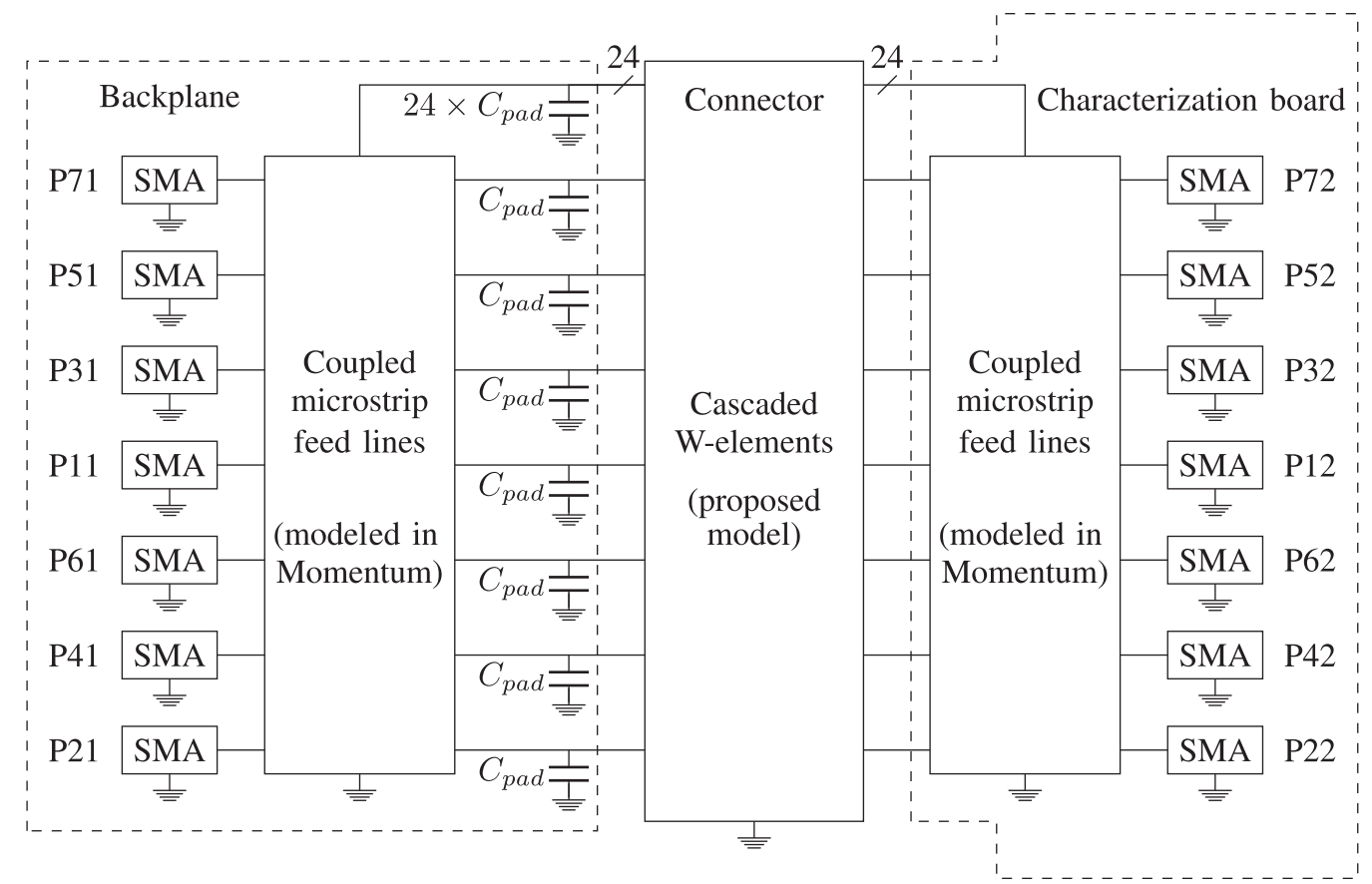

Fig. 10. Schematic representation of the model of the complete characterization setup that consists of the backplane (the SMA connectors, the coupled microstrip feed lines, and the capacitances of the pads), the proposed model of the connector (one W-element for the straight connector, four cascaded W-elements for the angled connector), and the characterization board (the coupled microstrip feed lines and the SMA connectors).

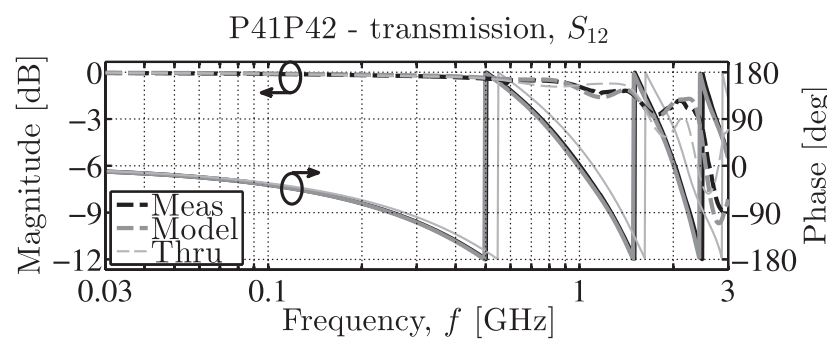

(a)

P41P31 - NEXT to the first neighbour

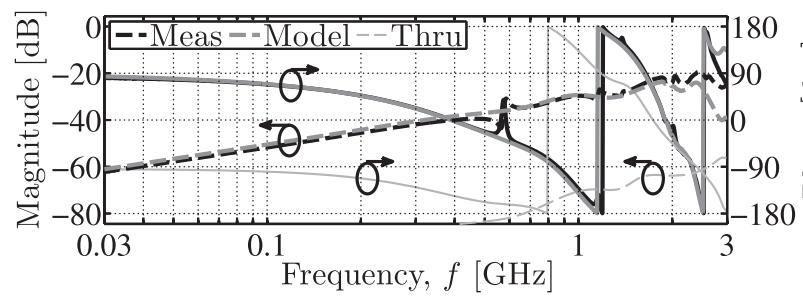

(c)

P41P52 - FEXT to the first neighbour

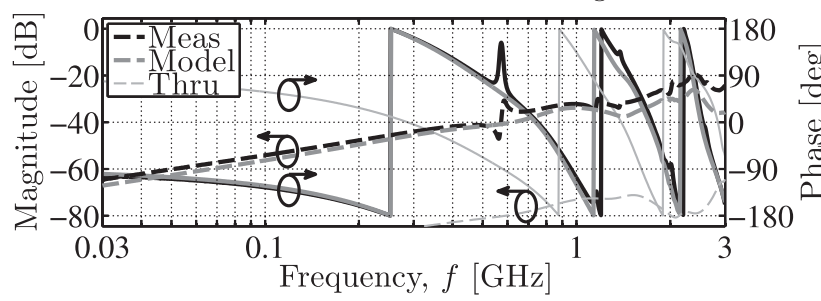

(e)
P41P42 - reflection, $S_{11}$

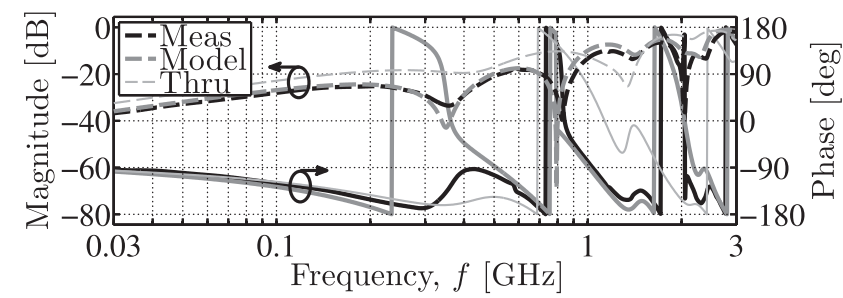

(b)

P41P21 - NEXT to the second neighbour

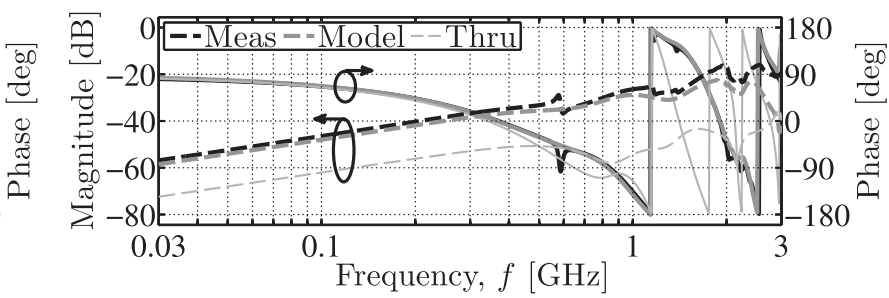

(d)

P41P62 - FEXT to the second neighbour

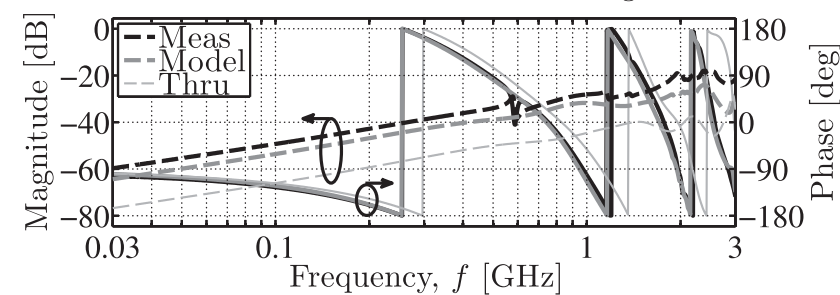

(f)

Fig. 11. Comparison of the measured and simulated $S$-parameters of the straight connector. (a) Transmission $S_{12}$. (b) Reflection $S_{11}$. (c) Crosstalk to the first neighbor on the backplane side (the NEXT). (d) Crosstalk to the second neighbor on the backplane side (the NEXT). (e) Crosstalk to the first neighbor on the characterization board side (the FEXT). (f) Crosstalk to the second neighbor on the characterization board side (the FEXT). Thinner and lighter line "Thru" is added to the graphs to emphasize the impact of the connector. It shows the characteristics of the model of the characterization setup when each pin of the connector is modeled by a short. 
P41P42 - transmission, $S_{12}$

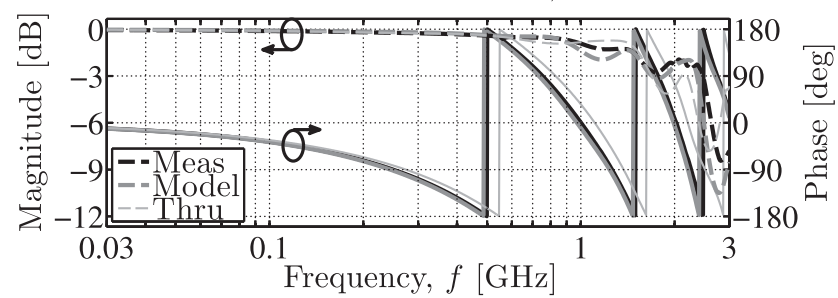

(a)

P41P31 - NEXT to the first neighbour

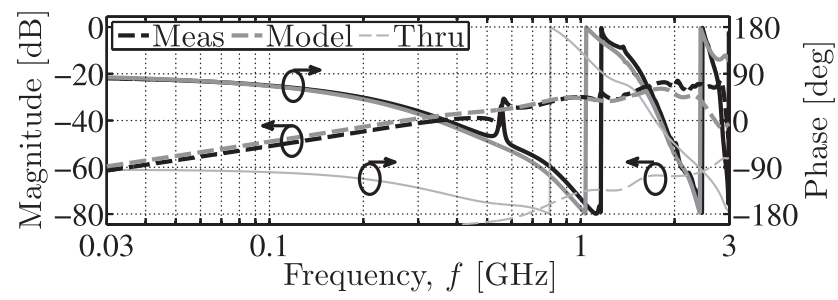

(c)

P41P52 - FEXT to the first neighbour

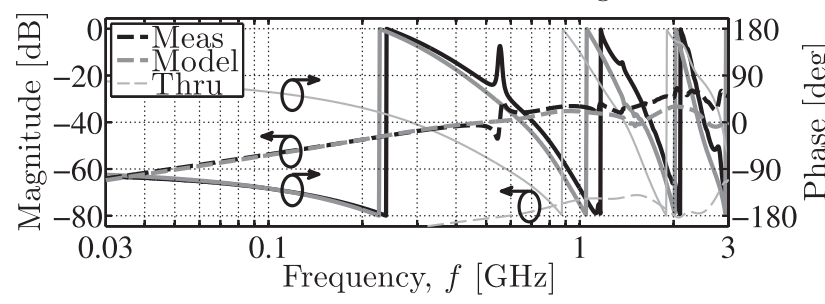

(e)

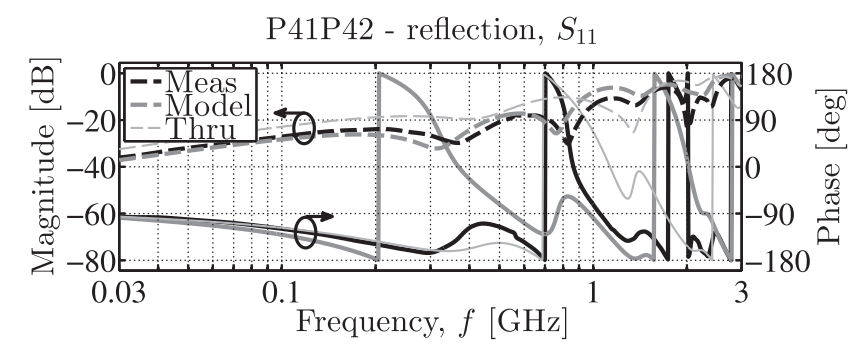

(b)

P41P21 - NEXT to the second neighbour

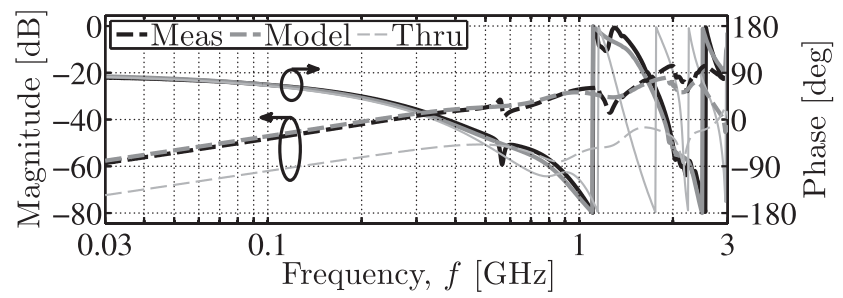

(d)

P41P62 - FEXT to the second neighbour

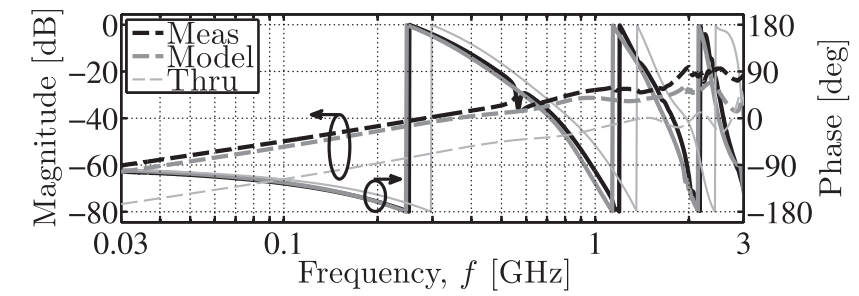

(f)

Fig. 12. Comparison of the measured and simulated $S$-parameters of the angled connector. (a) Transmission $S_{12}$. (b) Reflection $S_{11}$. (c) Crosstalk to the first neighbor on the backplane side (the NEXT). (d) Crosstalk to the second neighbor on the backplane side (the NEXT). (e) Crosstalk to the first neighbor on the characterization board side (the FEXT). (f) Crosstalk to the second neighbor on the characterization board side (the FEXT). Thinner and lighter line "Thru" is added to the graphs to emphasize the impact of the connector. It shows the characteristics of the model of the characterization setup when each pin of the connector is modeled by a short.

TABLE VI

RESULTS OF THE FSV

TECHNIQUE

\begin{tabular}{|c|c|c|c|}
\hline Structure & $\mathrm{ADM}$ & FDM & GDM \\
\hline Straight connector & $0.167(\mathrm{VG})$ & $0.291(\mathrm{G})$ & $0.265(\mathrm{G})$ \\
\hline Angled connector & $0.224(\mathrm{G})$ & $0.330(\mathrm{G})$ & $0.359(\mathrm{G})$ \\
\hline Calibration structures & 0.119 (VG) & $0.185(\mathrm{VG})$ & $0.186(\mathrm{VG})$ \\
\hline
\end{tabular}

changing features of the two sets, while combining ADM and FDM gives the global difference measure (GDM). The range of values for the ADM, FDM, and GDM is divided into six cat-egories, each with a language descriptor: excellent $(\mathrm{E})(<0.1)$, very good $(\mathrm{VG})$ $(0.1-0.2)$, good $(\mathrm{G})(0.2-0.4)$, fair $(\mathrm{F})(0.4-0.8)$, poor $(\mathrm{P})(0.8-1.6)$, and very poor (VP) $(>1.6)[28]-[30]$.

The ADM, FDM, and GDM are calculated for the magnitude in decibels and unwrapped phase of each characteristic shown in Fig. 11. The mean values of the ADM, FDM, and GDM are given in Table VI and they show good overall agreement of the model with the measurements. For reference, the FSV of the calibration structures is also added to Table VI and it shows very good agreement.

A resonance at approximately $600 \mathrm{MHz}$ can be seen in the measurements of both connectors and in all presented characteristics. Less pronounced resonances can also be seen at higher frequencies. These resonances are most likely the cavity-mode resonances [31] of the characterization board, formed between the second and third layers (see Fig. 9). Cavity-mode resonances can be suppressed by adding stitching vias that connect the two layers [32]. Stitching vias are used in the backplane and con-nector boards in [16] and [17] and no resonances are seen there.

\section{CONCLUSION}

Two 62-pin high-current card-edge connectors are modeled by a multisegment lossless multiconductor transmission line model. The calculation of the p.u.l. transmission line capaci-tance and inductance matrices of each uniform segment is based on the analytic expression for a generalized capacitance matrix of widely separated conductors. The calculated matrices are im-plemented in a circuit simulator by a W-element. The resulting circuit model is generated solely from the geometrical parame-ters of the connector without measurements or electromagnetic simulations being performed. The characterization setup is designed to validate the pro-posed model. It consists of two PCBs that are connected by the modeled connectors. The model of the two PCBs is generated by a combination of analytical and numerical meth-ods. The simulation of the complete characterization setup takes $5 \mathrm{~min}$. The simulation results are compared to the $S$-parameter measurements. The comparison of the transmission 
characteristics, reflection characteristics, NEXT and FEXT to the first and to the second neighbor up to $3 \mathrm{GHz}$ shows good overall agreement according to the FSV technique. The pro-posed modeling methodology enables fast analysis of EMC of complex systems connected by these multipin connectors. 
[1] L. Martens, High-Frequency Characterization of Electronic Packaging. Norwell, MA, USA: Kluwer, 1998.

[2] J. Li and J. Fan, "Radiation physics and design guidelines of high-speed connectors," IEEE Trans. Electromagn. Compat., vol. 58, no. 4, pp. 13311338, Aug. 2016.

[3] S. Sercu and L. Martens, "Experimental circuit model generation of nonuniform coupled multi-conductor structures," in Proc. ARFTG Conf. Dig.Spring, 1997, vol. 31, pp. 236-239.

[4] C.-H. Kao, C.-C. Tseng, F.-M. Lee, and M.-F. Lai, "A better technique using multisegment modeling and analysis of high-density and high-speed connectors," IEEE Trans. Adv. Packag., vol. 29, no. 1, pp. 140-148, Feb. 2006.

[5] H. Katzier, R. Reischl, and P. Pagnin, "SPICE-models for high-pincount board connectors," IEEE Trans. Compon., Packag., Manuf. Technol. B, vol. 19, no. 1, pp. 3-6, Feb. 1996.

[6] S. Pannala, A. Haridass, and M. Swaminathan, "Parameter extraction and electrical characterization of high density connector using time domain measurements," IEEE Trans. Adv. Packag., vol. 22, no. 1, pp. 32-39, Feb. 1999.

[7] K. Lee, H. K. Jung, J. Y. Sim, and H. J. Park, "Reduction of transient far-end crosstalk voltage and jitter in DIMM connectors for DRAM interface," IEEE Microw. Compon. Lett., vol. 19, no. 1, pp. 15-17, Jan. 2009.

[8] M.-S. Zhang, Y.-S. Li, L.-P. Li, and C. Jia, "Modeling and analyzing high-speed and high-density connectors by using multisegment multi-ple transmission lines model," IEEE Trans. Adv. Packag., vol. 31, no. 1, pp. 203-210, Feb. 2008.

[9] A. Deutsch et al., "Electrical characteristics of high-performance pin-insocket and pad-on-pad connectors," IEEE Trans. Compon., Packag., Manuf. Technol. B, vol. 20, no. 1, pp. 64-77, Feb. 1997.

[10] H. Hwangbo et al., "A scalable model of board to FPC interconnect using neural networks," in Proc. IEEE Int. Symp. Electromagn. Compat., Jul. 2007, pp. 1-7.

[11] E. McGibney and J. Barrett, "An overview of electrical characterization techniques and theory for IC packages and interconnects," IEEE Trans. Adv. Packag., vol. 29, no. 1, pp. 131-139, Feb. 2006.

[12] C. R. Paul, Analysis of Multiconductor Transmission Lines, 2nd ed. Hoboken, NJ, USA: Wiley, 2007.

[13] C. R. Paul and A. Feather, "Computation of the transmission line inductance and capacitance matrices from the generalized capacitance matrix," IEEE Trans. Electromagn. Compat., vol. EMC-18, no. 4, pp. 175-183, Nov. 1976.

[14] J. S. Savage and W. Smith, "Capacitance calculations for cable harnesses using the method of moments," IEEE Trans. Electromagn. Compat., vol. 37, no. 1, pp. 131-137, Feb. 1995.

[15] R. Blecic, N. Bako, R. Gillon, and A. Baric, "Broadband measurement of the magnetic moment normal to the printed circuit board plane using tem cell and phase information," in Proc. IEEE Int. Symp. Electromagn. Compat., Sep. 2013, pp. 955-960.

[16] R. Blecic, R. Gillon, B. Nauwelaers, and A. Baric, "EMC-oriented de-sign of output stage of synchronous buck converter," in Proc. IEEE Int. Workshop Electromagn. Compat. Integr. Circuits, Jul. 2017, pp. 91-96.

[17] R. Blecic, H. Stimac, R. Gillon, B. Nauwelaers, and A. Baric, "Improved estimation of radiated fields of unintentional radiators by correction of the impedance mismatch between a transverse electromagnetic cell and a hybrid coupler," IEEE Trans. Electromagn. Compat., to be published.

[18] TE Connectivity, Card-edge connector, 6-5530843-5 product details, Feb. 2013. [Online]. Available: http://www.te.com/usa-en/product-6-55308435.html

[19] TE Connectivity, Card-edge connector, 5532600-4 product details, Feb. 2013. [Online]. Available: http://www.te.com/usa-en/product-55326004.html

[20] HSPICE User Guide: Signal Integrity, Synopsys, 2010.

[21] T. Mandic, R. Gillon, B. Nauwelaers, and A. Baric, "Characterizing the TEM cell electric and magnetic field coupling to PCB transmission lines," IEEE Trans. Electromagn. Compat., vol. 54, no. 5, pp. 976-985, Oct. 2012.
[22] N. K. Das, S. M. Voda, and D. M. Pozar, "Two methods for the measurement of substrate dielectric constant," IEEE Trans. Microw. Theory Technol., vol. 35, no. 7, pp. 636-642, Jul. 1987.

[23] Advanced Design System Documentation Set, Keysight Technologies, Santa Rosa, CA, USA, 2012.

[24] Integrated Circuits Measurement of Electromagnetic Emissions, $150 \mathrm{kHz}$ to $1 \mathrm{GHz}$-Part 2: Measurement of Radiated Emissions, TEM Cell and Wideband TEM-Cell Method, IEC Standard 61967-2:2005, 2005.

[25] Y. Zhang, J. Fan, G. Selli, M. Cocchini, and F. de Paulis, "Analytical evaluation of via-plate capacitance for multilayer printed circuit boards and packages," IEEE Trans. Microw. Theory Technol., vol. 56, no. 9, pp. $2118-2128$, Sep. 2008.

[26] EMPro 2015, FEM Simulation, Keysight Technologies, Santa Rosa, CA, USA, 2015.

[27] K. Vaz, K. M. Ho, and M. Caggiano, "Error reducing techniques for the scattering parameter characterization of differential networks using a two-port network analyzer," in Proc. 28th Int. Spring Seminar Electron. Technol., Meeting Challenges Electron. Technol. Progress, 2005, pp. 342-347.

[28] IEEE Recommended Practice for Validation of Computational Electromagnetics Computer Modeling and Simulations, IEEE Std 1597.1, 2008.

[29] A. P. Duffy, A. J. M. Martin, A. Orlandi, G. Antonini, T. M. Benson, and M. S. Woolfson, "Feature selective validation (FSV) for validation of computational electromagnetics (CEM) - Part I: The FSV method," IEEE Trans. Electromagn. Compat., vol. 48, no. 3, pp. 449-459, Aug. 2006.

[30] A. Orlandi, A. P. Duffy, B. Archambeault, G. Antonini, D. E. Coleby, and S. Connor, "Feature selective validation (FSV) for validation of computational electromagnetics (CEM) - Part II: Assessment of FSV performance," IEEE Trans. Electromagn. Compat., vol. 48, no. 3, pp. 460-467, Aug. 2006.

[31] M. Leone, "The radiation of a rectangular power-bus structure at multiple cavity-mode resonances," IEEE Trans. Electromagn. Compat., vol. 45, no. 3, pp. 486-492, Aug. 2003.

[32] K. B. Wu, R. B. Wu, and D. D. Zutter, "Modeling and optimal design of shorting vias to suppress radiated emission in high-speed alternating pcb planes," IEEE Trans. Compon., Packag., Manuf. Technol., vol. 1, no. 4, pp. 566-573, Apr. 2011. 
Raul Blecic (S'10) was born in Rijeka, Croatia, in 1986. He received the Dipl.-Ing. degree in electrical engineering from the University of Zagreb, Zagreb, Croatia, in 2009. He received the double Ph.D. degree in electrical engineering from the University of Zagreb and Katholieke Universiteit Leuven, Leuven, Belgium, in 2018.

His research interests include electromagnetic compatibility of integrated circuits, integrated circuit design, and modeling of microwave structures and components.

Niko Bako received the Dipl.-Ing. and the Ph.D. de-grees in electrical engineering from the University of Zagreb, Zagreb, Croatia, in 2006 and 2016, respec-tively.

From 2006 to 2010, he was with Systemcom Ltd., Zagreb, Croatia, where he worked on low power front-ends. From 2010 to 2016, he was with the Uni-versity of Zagreb. Since 2016, he is with Texas Instruments Deutschland. His research interests include low-power data converters, oscillators, PLLs, and op-erational amplifiers.

Renaud Gillon (S'89-M'92) was born in Brussels, Belgium, in 1968. He received the M.Sc. and Ph.D. degrees in electrical engineering from the Universite catholique de Louvain, Louvain-la-Neuve, Belgium, in 1991 and 1998, respectively.

In 1998, he joined ON Semiconductor Belgium (ONSEMI), Oudenaarde, Belgium, and then Alcatel Microelectronics as a Modeling Engineer and man-aged the team from 2000 to early 2006, taking an active role in the development of design kits for the company's RF and smart-power processes. He is currently responsible for several research and innovation programs with ONSEMI, in the field of electrostatic discharge, electromagnetic compatibility (EMC), and power. He has authored or coauthored more than 60 publications. His current research interests include modeling of HV and RF components, RF measure-ment techniques, electro-thermal simulation, and predictive simulation of EMC tests at the IC level.

Adrijan Baric (M'98-SM'16) received the Dipl.-Ing. and M.Sc. degrees in electrical engineering from the University of Zagreb, Zagreb, Croatia, in 1982 and 1985, respectively, and the Ph.D. degree in electronics from the Dublin City University, Dublin, Ire-land, in 1995.

Since 1984, he has been with the University of Za-greb, where he is currently a Professor. His research interests include semiconductor device modeling, in-tegrated circuit design, interconnect modeling, and electromagnetic compatibility.

Bart Nauwelaers (S'79-M'88-SM'99) was born in Niel, Belgium on July 7, 1958. He received the M.S. and $\mathrm{Ph} . \mathrm{D}$. degrees in electrical engineering from the Katholieke Universiteit Leuven (KU Leuven), Leuven, Belgium, in 1981 and 1988, respectively, and the Master's degree in design of telecommunication systems from ParisTech Télécom, Paris, France, in 1992.

Since 1981, he has been with the Department of Electrical Engineering, KU Leuven. He teaches courses on microwave engineering, analog and dig-ital communications, wireless communications, and design in electronics and telecommunications. For the last 20 years, he has served education in several functions, the last one being the Program Director for the bachelor and master programs in electrical engineering. His research interests include microwave antennas, passive components, interconnects, microwave integrated circuits, monolithic microwave integrated circuits, linear and nonlinear device modeling, microelectromechanical systems, and wireless communications.

Dr. Nauwelaers is a former Chair of the IEEE ANTENNAS AND PROPAGA-TION/MICROWAVE THEORY and Techniques Benelux Section and a past Chair of the URSI-Benelux. 